\title{
XXV. Über einige bemerkenswerte Zwillinge von Atacamit und einige andere Kupfermineralien von Collahurasi, Provinz Tarapacá, Chile.
}

\author{
Von \\ W. E. Ford in New Haven, Connecticut. \\ Übersetzt von W. Brendler. \\ (Mit 12 Textfiguren.)
}

Vor einiger Zeit wurde der Brush-Sammlung von Herrn Ernst Schernikow in New York City eine Suite von Kupfermineralien, welche von Collahurasi, Chile, stammten, zum Geschenk gemacht. Dieselbe enthielt u. a. folgende selteneren Mineralien: Atacamit, Brochantit, Olivenit, Klinoklas und Konichalcit. Atacamit und Brochantit kamen dabei in Krystallen und beträchtlicher Menge vor, sodaß sich eine ausgezeichnete Gelegenheit zu näherer Untersuchung darbot.

\section{Atacamit.}

Dieses Mineral wurde in krystallinischen Massen gefunden, welche zumeist aus winzigen Kryställchen bestehen; bisweilen kommen jedoch Einzelkrystalle von 4 bis $5 \mathbf{m m}$ Länge vor. Diese Krystalle erwiesen sich bei der Untersuchung als ungewöhnlich interessant.

Es waren daran nur wenige Formen vorhanden, nämlich: $m\{110\}$, $x\{140\}, b\{010\}, e\{011\}, r\{111\}$ und $n\{121\}$. Die Mehrzahl der Krystalle zeigte - wie Fig. 1 erkennen läßt - nur das Brachypinakoid $b$, das Grundprisma $m$ und das Doma e. Die Pyramiden $r$ und $n$ und das Prisma $x$ konnten nur an wenigen Krystallen und auch dann nur als schmale Abstumpfungen beobachtet werden, wie Fig. 2 zeigt. Das Interẹssante an diesen Krystallen ist jedoch die gleichförmig wiederkehrende und ungewöhnliche $\mathrm{Zwillingsbildung,} \mathrm{welche} \mathrm{sie} \mathrm{zeigen.} \mathrm{Das} \mathrm{betr.} \mathrm{Zwillings-}$ gesetz ist für den Atacamit neu und erwies sich manchmal als recht compliciert und schwierig zu deuten, aber die gute l̈bereinstimmung zwischen 
den gemessenen und den berechneten Winkelwerten läßt, wie die im nachstehenden aufgeführten Tabellen zeigen, keinen Zweifel daran, daß es richtig abgeleitet worden ist.

Fig. 1.

Fig. 2.

Fig. 3.
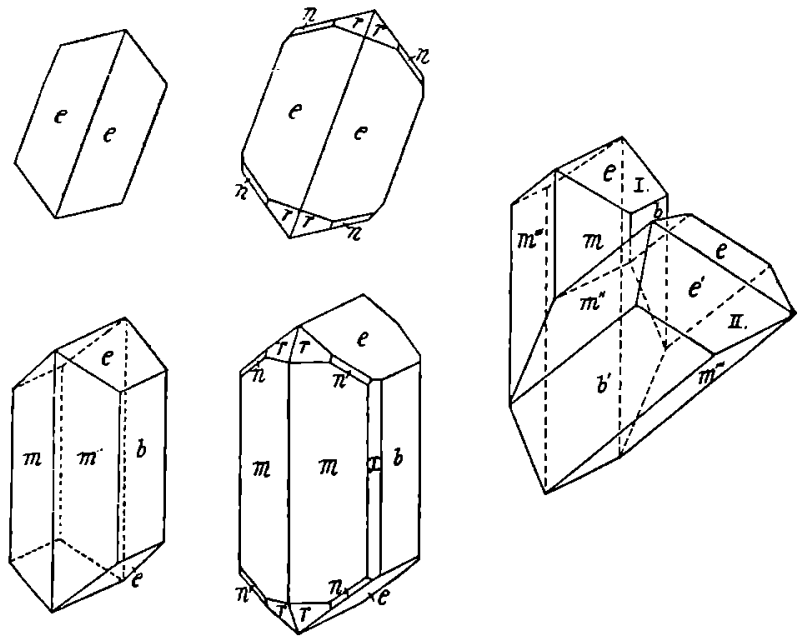

Das Zwillingsgesetz kann man folgendermaßen formulieren: Eine der e-Fläehen des in Zwillingsstellung befindlichen Krystalls ist immer einer der $e$-Flächen des in Normalstellung befindlichen Individuums parallel. Dies ist bei einer Betrachtung der Krystalle leicht ersichtlich, denn eine Brachydomenfläche des verzwillingten Individuums spiegelt immer zusammen mit einer Domenfläche des in Normalstellung befindlichen Krystalls ein. Ferner erscheint der in Zwillingsstellung befindliche Krystall um die Normale auf $c\{011\}$ als $Z$ willingsaxe um einen solchen Winkel gedreht, daß die zweite $e$-Fläche des verzwillingten Krystalls in die Prismenzone des in Normalstellung befindlichen Individuums zu liegen kommt.

Diese Beziehungen lassen sich am besten an der Hand von Fig. 3 erläutern, welche keine Abbildung eines wirklichen Krystalls darstellt, sondern lediglich zur Klarlegung des Zwillingsgesetzes dienen soll. Die Fläche $e$ des in Zwillingsstellung befindlichen Krystalls (Nr. II) ist parallel $e$ des in Normalstellung befindlichen Individuums (Nr. I), während $e^{\prime}$ von $\mathrm{Nr}$. II mit den Prismen- und Pinakoidflächen des vertical gestellten. Krystalls in eine Zone fält. Dies konnte an den Zwillingskrystallen bei den Messungen am Reflexionsgoniometer mit Sicherheit nachgewiesen werden. Die Reflexe, welche die $e$-Flächen der beiden Individuen gaben, fielen zusammen, und derjenige von $e^{\prime}$ von Nr. II lag mit denen der Prismenflächen von Nr. I in ein und derselben Zone. Dies fand sich an allen gemessenen Krystallen bestätigt 
mit so geringen Abweichungen, wie sie durch die bisweilen ziemlich mittelmäßige Beschaffenbeit der Flächen leicht hervorgerufen werden können. Die zwischen den beiden Individuen bestehenden Beziehungen zeigt die stereographische Projection, Fig. 4.

Fig. 4.

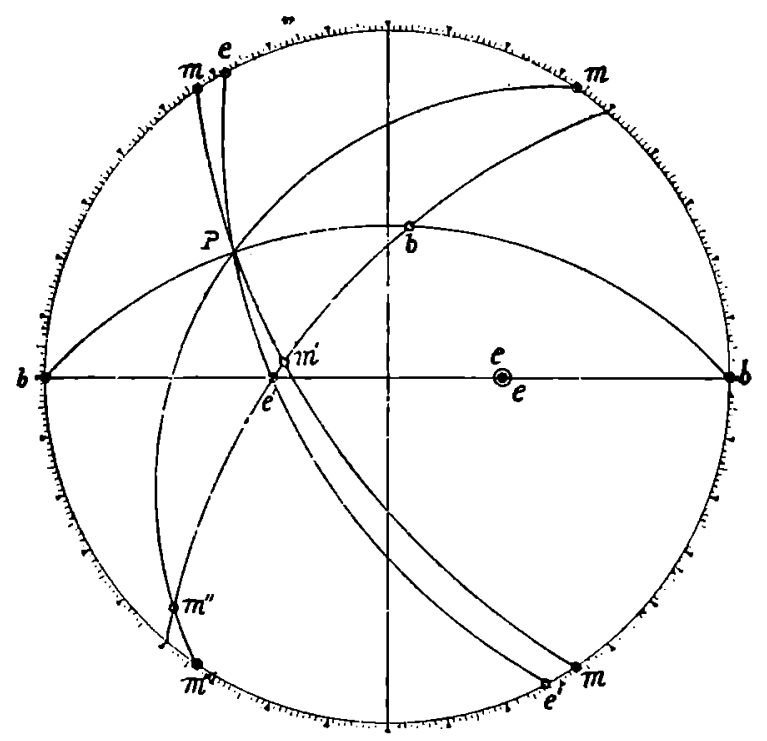

Um die beiden Krystallindividuen in ihre betr. Stellungen zu bringen, z. B. $e^{\prime}$ von Nr. II in die Prismenzone von Nr. I, müssen wir uns den Krystall Nr. II um die Normale auf $e\{011\}$ als Axe um 112040' gedreht denken. Diese Tatsache macht die vorliegenden $Z$ willinge zu ungewöhnlichen und schwer zu deutenden, denn der Drehungswinkel des einen Individuums um die $Z$ willingsaxe beträgt für gewühnlich $180^{\circ}$ oder bisweilen auch $120^{\circ}$.

In $A$ nbetracht dieser Eigentümlichkeit können wir die Fläche $e$, obschon sie beiden Individuen gemeinsam ist, weder als Zwillingsfläche ansehen wenigstens nicht im gewöhnlichen Sinne --, noch ihre Normale als Zwillingsaxe.

Die Fläche, deren $\mathrm{Pol}$ in der stereographischen Projection mit $P$ bezeichnet ist und welche in Fig. 3 die Verwachsungsfäche der beiden Krystallindividuen darstellt, würde der im allgemeinen üblichen Definition einer Zwillingsebene entsprechen. Die in Zwillingsstellung befindliche Hälfte des Krystalls ließe sich - wie die stereographische Projection erkennen läßt - durch Spiegelung der normalen Hälfte an dieser Ebene erhalten; denn auf einem größten Kreise, der von irgend einer Fläche des Krystalls I durch den $P$ ol $P$ gezogen wird, läßt sich auf der anderen Seite von $P$ in 
der gleichen Winkeldistanz die entsprechende Fläche des in $\mathrm{Z}$ willingsstellung befindlichen Individuums Nr. II auffinden. Diese Fläche $P$ stimmt jedoch bezüglich ihrer Lage mit keiner der am Atacamit vorkommenden Flächen überein, am nüchsten liegt sie noch der müglichen Pyramide $\{\overline{4} \overline{7} 5\}$. Zur Prüfung der Richtigkeit des hier aufgestellten Zwillingsgesetzes sind in den nachfolgenden Tabellen die gemessenen und berechneten Winkelwerte nebeneinandergestellt.

Die Messungen der Krystalle erfolgten sowohl am zweikreisigen wie am einkreisigen Goniometer und gaben folgende Werte:

1) Winkelmessungen am zweikreisigen Goniometer zur Feststellung der Lage der Flächen des verzwillingten Individuums (Nr. II).

Flächen von $\mathrm{Nr}$. II.

e

$e^{\prime}$

$m$

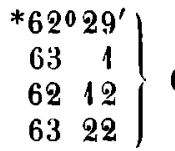

*8 59

$8 \quad 44$

1138

121

$m^{\prime} \quad * 47 \quad 45$

4738

4525

$\begin{array}{lll}46 & 20\end{array}$

$46 \quad 31$

$\begin{array}{ll}46 & 3\end{array}$

$46 \quad 12$

b

1020

*80 48

8029

\section{ssen.}

e Mittel:

*36058'

3650

$3716\} 36050$

$36 \quad 45$

$\begin{array}{ll}36 & 24\end{array}$

*90 1

$90 \quad 38$

\begin{tabular}{ll|llllll}
90 & 21 & 90 & 6 & 62 & 25 & 90 & 0
\end{tabular}

8924

*34 31

3144

$34 \quad 1$

3321

3324

$\begin{array}{llll}847 & 33 & 57\end{array}$

*83 56

848

$83 \quad 46$

8258

852

81. 54

842

48 3. $\overbrace{\varphi}^{\text {Berechnet. }}$

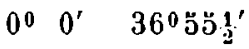

8 4 3357

2) Winkelmessungen am cinkreisigen Goniometer zwischen Flächen der Krystallindividuen I und II.

Gemessen: Miltel: Berechnet:

$e^{\prime}$ von $\mathrm{Nr} . \mathrm{I}: e^{\prime}$ von $\mathrm{Nr}$. II

$106024^{\prime}$

$10 \ddot{3} 54$

$10623 \int^{100^{\circ} 11^{\prime}} 106010^{\prime}$

$106 \quad 3$

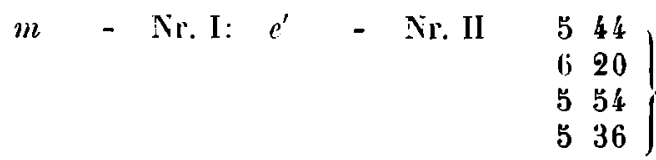

5ั 53

5ั :391. 


\begin{tabular}{|c|c|c|c|c|c|c|c|c|}
\hline & & & & & & Gemessen: & Mittel: & Berechnet: \\
\hline$m^{\prime \prime \prime}$ & von & Nr. I: & $m^{\prime \prime}$ & von & Nr. II & $\left.\begin{array}{ll}10^{0} & 39^{\prime} \\
11 & 17 \\
10 & 42 \\
11 & 17\end{array}\right\}$ & $1101^{\prime}$ & $10^{0} 57^{\prime}$ \\
\hline$m$ & - & Nr. I: & $m^{\prime \prime}$ & - & Nr. II & $\left.\begin{array}{cc}67 & 56 \frac{1}{2} \\
68 & 1\end{array}\right\}$ & 6758 & 6754 \\
\hline$m^{\prime \prime \prime}$ & - & Nr. I: & $m^{\prime}$ & - & Nr. II & $\left.\begin{array}{rr}76 & 56 \\
76 & 6 \\
76 & 59 \\
76 & 50\end{array}\right\}$ & $76 \quad 42$ & $76 \quad 29$ \\
\hline
\end{tabular}

In vorstehenden Tabellen sind sämtliche an den verschiedenen Krystallen gemessene Winkeln aufgenommen worden ohne Rücksicht auf die Beschaffenheit der Flächen und das daraus zu folgernde Gewicht der einzelnen Winkelmessungen. Im allgemeinen stimmen die Mittelwerte der Winkel der verschiedenen Serien mit den berechneten Winkeln ziemlich gut überein. In der Tabelle der mit dem zweikreisigen Goniometer erhaltenen Winkelwerte ist der durch Messung des am besten entwickelten Krystalls erhaltene Wert, sowie derjenige, welcher die genaueste Justierung am Goniometer gestattete, in jeder Reihe an erster Stelle aufgeführt und weiterhin durch einen Stern * kenntlich gemacht.

Die Übereinstimmung zwischen diesen Winkeln und den entsprechenden theoretischen Werten ist ziemlich genau. Die größte Abweichung zwischen den gemessenen und den berechneten Winkeln zeigt sich bei den Flächen der Prismenzone und zwar besonders bei den $\varphi$-Winkeln. Dieses Ergebnis war bei der gewöhnlich ziemlich schlechten Beschaffenheit der Prismenflächen und der häufig an denselben auftretenden verticalen Streifung von vornherein zu erwarten.

Bei den Messungen am einkreisigen Goniometer ist, wie man bemerken kann, die Übereinstimmung zwischen den gemessenen und den berechneten Winkeln größer und im allgemeinen sehr befriedigend. Dies ließ sich voraussehen, da bei diesen kleinen Krystallen, deren Flächenbeschaffenheit meist nicht die beste war, die Justierung des Krystalls am zweikreisigen Goniometer schwierig ist und bestenfalls nur eine angenäherte sein kann, sodaß infolgedessen die damit gemessenen Winkel kein so großes Gewicht haben wie die am einkreisigen Instrument erhaltenen, bei welchem für jede Messung extra justiert wird. Betrachtet man jedoch die Messungen in ihrer Ge. samtheit, so ist die Übereinstimmung mit den theoretischen Winkelwerten genügend genau, um ohne $\mathrm{Zweifel} \mathrm{nachzuweisen,} \mathrm{daß} \mathrm{das} \mathrm{Zwillingsgesetz}$ richtig aufgestellt worden ist.

Dem Verfasser ist kein einziger Fall bekannt, in welchem eine der im vorstehenden erörterten vollkommen analoge $Z$ willingsverwachsung aufgetreten wäre, und er vermag keine ausreichende Erklärung oder Theorie zu 
geben, welche den bei diesen $\mathbf{Z}$ willingen beobachteten Eigentümlichkeiten völlig gerecht werden könnte. Es ist eine bemerkenswerte und vielleicht bezeichnende Tatsache, daß die Flächen des in Zwillingsstellung befindlichen Krystalls Nr. II, zum gröBten Teil wenigstens innerhalb einiger Grade, den Flächen des in Normalstellung befindlichen Individuums Nr. I nahe liegen. Diese Verhältnisse bringt die stereographische Projection, Fig. 4, klar zur Anschauung; in derselben liegt $e^{\prime}$ von Nr. II nahe bei $m$ von Nr. I; $m^{\prime \prime}$ von II bei $n^{\prime \prime \prime}$ von I; $m^{\prime}$ von II bei $e^{\prime}$ von I, und $b^{\prime}$ von II fällt nahezu in die Makrodomenzone von I. Es müchte scheinen, als ob hier ein Fall von Zwillingsverwachsung vorläge, bei welchem die Stellung des verzwillingten Individuums vielmehr durch den Parallelismus einer bevorzugten Fläche und durch zonale Beziehungen zwischen den beiden Individuen kontrolliert werden künnte, als durch das Vorhandensein einer gewühnlichen Zwillingsebenc oder $A x e$.

Dic Fläche $P$, welche - falls sie eine mügliche Krystallfläche wäre als Zwillingsebene zu bezeichnen sein würde, kann man in ähnlicher Weise als Zusammensetzungs- oder Verwachsungsfläche ansehen, wie den srhombischen Schnilt« bei den Plagioklasen, wenn dieselben nach dem Periklingesetz verzwillingt sind.

Daß nicht alle regelmäßigen Verwachsungen von Krystallen den gewöhnlichen Zwillingsgesetzen gehorchen, hat man vor einiger Zeit erkannt ${ }^{1}$ ). Diese Verwachsungen sind keine Zufallsproducte, sondern sie folgen Gesetzen, welche sich genau formulieren lassen; sie werden öfters an Stücken verșchiedenen Vorkommens gefunden und sollten in jeder vollständigen Betrachtung der Z $Z$ willingsverwachsungen Aufnahme finden. Goldschmidt hat kürzlich versucht, diese ungewöhnlichen Verwachsungen in eine neue Classification der Zwillingsverwachsungen mit aufzunehmen und hat dem von ihm aufgestellten neuen Gruppen verschiedene Namen beigelegt, wie \Heterozwillinge «, \Einflächige Verwachsung «, \Einzonige Verwachsung ${ }^{2}$ ). Es steht zu erwarten, daß noch mehr derartige Beispiele entdeckt und noch andere Gruppen aufgestellt werden, nachdem einmal die Aufmerksamkeit auf diese $\mathrm{Zwillinge} \mathrm{niederer} \mathrm{Symmetrie} \mathrm{hingelenkt} \mathrm{worden} \mathrm{ist.} \mathrm{Tat-}$ sächlich scheinen die hier beschriebenen $Z$ willinge keiner der in Goldschmidt's Aufstellung erwähnten Gruppen genau zu entsprechen, am nächsten würden sie noch der Gruppe der »Helerozwillinge « stehen. Ein kürzlich von $\mathrm{Paul}$ und Goldschmidt ${ }^{3}$ ) beschriebener Heterozwilling von Orthoklas ähnelt in mancher Hinsicht den vorliegenden Atacamitzwillingen. Das an dem Orthoklaszwilling herrschende Gesetz lïßt sich folgendermaßen

1) V. Goldschmidt, diese Zcitschr. 1898, 30, 254; 1907, 43, 347.

2) Diese Zeitschr. 1907, 43, 582.

3) Diese Zeitschr. 1909, 45, 471. 
formulieren: Fläche $c\{001\}$ von Nr. II ist der Fläche $b\{010\}$ von Nr. I parallel; die Zone $c(001)-m(110)$ von Nr. II fällt mit der Zone $b(010)-$ $c(001)$ von Nr. I zusammen, und die Fläche $b\{010\}$ von Nr. II kommt in die Zone $c(001)-y(201)$ von Nr. I zu liegen. Es tritt hier also der Fall ein, $d a ß$ an den beiden Individuen zwei ungleichartige Flächen Parallelismus zeigen, daß ferner zwei ungleiche Zonen zusammenfallen, und daß schließlich eine wesentliche Fläche des einen Individuums in eine wichtige Zone des anderen fällt. Andererseits können wir an den Atacamitzwillingen beobachten, daß sich zwei ähnliche Flächen in Parallelstellung befinden, daß eine charakteristische Fläche von Nr. II in einer wichtigen Zone von Nr. I liegt, und daß, ganz im allgemeinen, die Flächen von Nr. Il in der Nähe der Flächen oder Zonen von Nr. I liegen.

liig. : :.

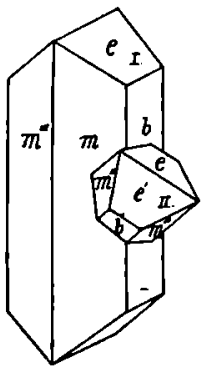

Fig. 6.

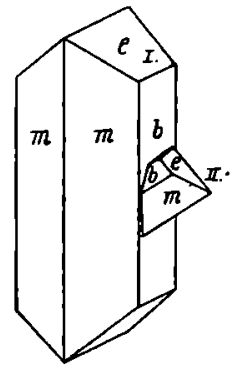

Fig. 7.

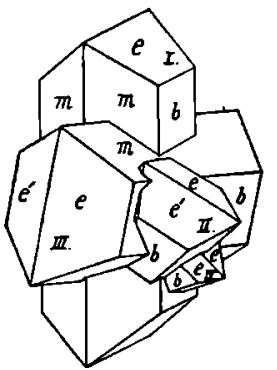

Die Zwillingskrystalle treten in zweierlei Typen auf. Die erste derselben stell, wie die Figg. 5 bis 7 erkennen lassen, einen Durchwachsungszwilling dar, bei welchem das eine Individuum durch das andere hindurchgewachsen bezw. aus ihm herausgewachsen ist. Bei dieser Art von Zwillingen ist der eine Krystall gewöhnlich viel größer als der andere ausgebildet, und der kleinere zeigt, aus der Seite des ersteren hervorragend, nur die Flächen des einen Endes in freier Entwicklung. Fig. 5 stellt einen derartigen Krystall dar. Eine solche Verwachsung kann zwejerlei Art sein, nämlich je nachdem ob das verzwillingte Individuum bezüglich des in Normalstellung befindlichen nach vorn oder nach hinten gewendet erscheint. Diese Zwillinge bilden bisweilen sehr zusammengesetzte Gruppen, derart, daß ein zweites Individuum sich zum ersten in Zwillingsstellung befindet, wïhrend ein drittes wieder hinsichtlich der zweiten Zwillingsstellung einnimmt usw.

Eine derartige complicierte Verwachsung ist in Fig. 7 dargestellt; Krystall Nr. II befindet sich dabei hinsichtlich Nr. I in Zwillingsstellung, während Nr. III gegenüber Nr. II Zwillingsstellung einnimmt, sodaß Fäche $e$. von III der Fläche $e^{\prime}$ von II parallel ist usw.

Die zweite Art der Zwillingskrystalle zeigt, obschon dem gleichen 
Gesetz folgend, ein durchaus anderes Aussehen. Sie besteht aus einem normalen Individuum, das ein mehr gedrungenes Wachstum zeigt und dessen Domenflächen an beiden Enden nahezu, wenn nicht völlig von vier zu ihm in Zwillingsstellung befindlichen Krystallen bedeckt sind.

Fig. 8.

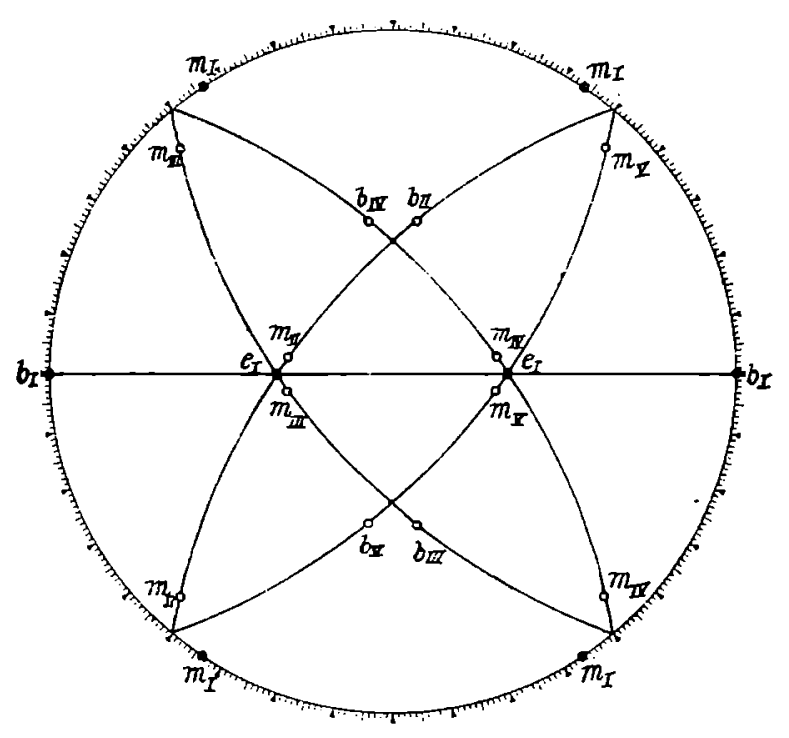

Die $e$-Flächen von je zweien dieser in Zwillingsstellung befindlichen Individuen verlaufen parallel einer der Flächen des normalen Krystalls, wobei der eine $Z$ willing nach vorn, der andere nach hinten gewendet ist. Die anderen beiden der in $Z$ willingsstellung befindlichen Individuen zeigen ähnliche Anordnung und Beziehungen mit der Ausnahme, daß eine ihrer $e$-Flächen der zweiten $e$-Fläche des normalen Krystalls parallel geht. An diesen in $Z$ willingsstellung befindlichen Krystallen sind für gewöhnlich nur die Flächen des Prismas und des Brachypinakoids entwickelt, welche an der Spitze des normalen Krystalls seichte Rinnen bilden, die beiderseits oberhalb seiner Domenflächen in der Richtung der Brachydiagonale verlaufen. Gelegentlich finden sich an den in Zwillingsstellung befindlichen Individuen auch $e$-Flächen, welche entweder zur Entstehung seitlicher Ausladungen über den $b\{100\}$-Flächen des normalen Krystalls Veranlassung geben, oder bisweilen auch wenig einspringende Vinkel längs der Spitze des Krystalls bilden. Iiese Beziehungen sind in der stereographischen Projection, Fig. 8, dargestellt, welche die Formen des in Normalstellung befindlichen Krystalls I, wie auch die Prismen- und Pinakoidflächen der vier verschiedenen in Zwillingsstellung befindlichen Individuen II, III, IV 
und $\mathrm{V}$ enthält. Einen Krystall dieser Ausbildungsweise zeigt Fig. 9, wobei die vier Zwillingsindividuen die Domenflächen des normalen Krystalls nicht vollständig bedecken. Diese Ảbbildung läßt auch die den verzwillingten Individuen IV und $\mathrm{V}$ gemeinsame $e$-Fläche erkennen, welche oberhalb der $b$-Fläche des normalen Krystalls liegt. Bisweilen hüllen die verzwillingten Individuen die Enden des in Normalstellung befindlichen Krystalls vollständig ein, sodaß von lelzterem nur noch die Flächen seiner Prismenzone sichtbar sind. Fig. 10 zeigt einen derartigen an beiden Enden ausgebildeten Krystall. In Fig. 11 ist der gleiche Krystall noch einmal gezeichnet mit dem Unterschiede, daß die in Fig. 10 auf der Rückseite liegenden Linien ausgezogen wurden, um die Übersicht über diese merkwürdigen Zwillingsbildungen zu erleichtern. Außer den hier abgebildeten Zwillingsgruppen kommen noch viel compliciertere vor, bei welchen beide Typen von Zwillingen in höchst verworrener Weise miteinander combiniert sind.

Fig. 9.

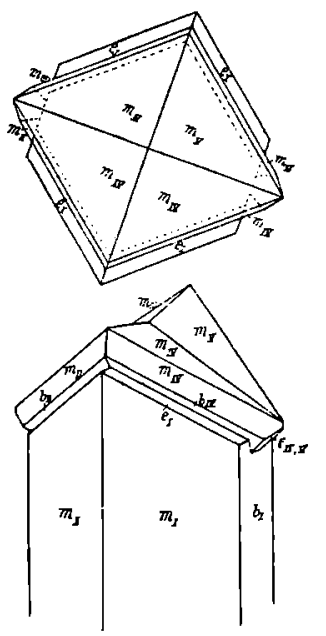

Fig. 10.

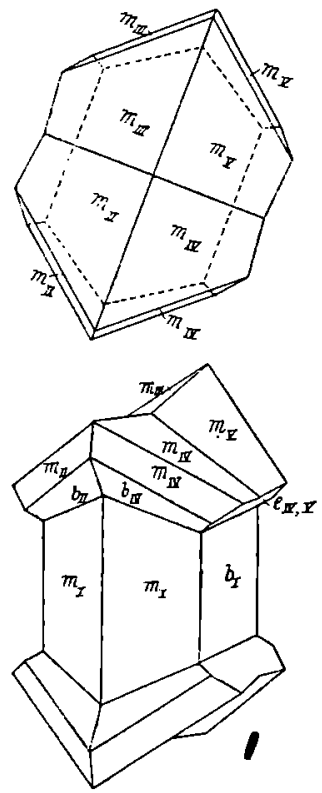

Fig. 11.

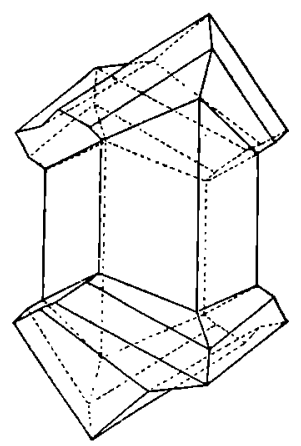

Eine am vorliegenden Atacamit ausgeführte Analyse stimmte mit der theoretischen Zusammensetzung sehr gut überein, wie nachstehende Aufstellung erkennen läßt:

\begin{tabular}{lrr}
$\mathrm{Cl}$ & $\mathbf{1 6 , 5 5}$ & 16,6 \\
$\mathrm{Cu}$ & 14,82 & 14,9 \\
$\mathrm{CuO}$ & 56,01 & 55,8 \\
$\mathrm{H}_{2} \mathrm{O}$ & $\mathbf{1 2 , 6 9}$ & 12,7 \\
\cline { 2 - 2 } & 100,07 & 100,0
\end{tabular}


Brochantit. Brochantit war dasjenige Mineral, welches auf den Stufen am häufigsten beobachtet wurde. Er kommt meist in dünnen prismatischen Krystallen vor, welche - einander durchkreuzend und durchspießend - kürnig-krystallinische Massen bilden. Zufällig wurde ein Krystall mit gutentwickelten Flächen entdeckt, der Messungen vorzunehmen gestattete. Es ließen sich daran folgende Formen feststellen: $b\{010\}, m\{110\}, r\{120\}$ und $v\{101\}$; Fig. 12 zeigt ihre charakteristische Ausbildungsweise. Neben dieser Art des Vorkommens wurde Brochantit noch in sehr dünnen, meist haarförmigen prisinatischen Krystallen beobachtet, welche auf der Oberfläche des Ganggesteins in radialfaserigen Büscheln angeordnet sind. Infolge ihrer durchscheinenden leuchtendgrünen Farbe bildet diese Varietät außerordentlich prächtige Schaustücke. Eine quantitative Analyse dieser dünnen Krystallnadeln wurde soweit durchgeführt als zur Feststellung der Identität mit Brochantit erforderlich war. Das untersuchte Material erwies sich als ideal rein und die erhaltenen Analysenresultate stimmen infolgedessen mit den theoretischen Werten sehr gut überein, wie nachstehende Zusammenstellung zeigt:

$\begin{array}{lcccc} & \text { Mi. } & \text { II. } & \text { Millel: } & \text { Berechnet: } \\ \mathrm{CuO} & 70,41 & 70,16 & 70,29 & 70,37 \\ \mathrm{SO}_{3} & 17, \mathrm{31} & 17,59 & 17,54 & 17,69 \\ \mathrm{H}_{2} \mathrm{O} & 11,91 & 19,01 & 11,96 & 11,94 \\ & & & 99,79 & 100,00\end{array}$

Fig. 12.

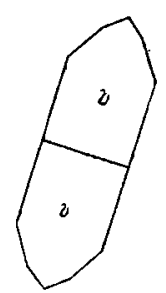

Olivenit kommt auf den Stufen in dünnen, spießigen, prismatischen Krystallen von licht- bis dunkel-olivengrüner Farbe vor. Das Mineral gab die für Olivenit charakteristischen Reactionen, und es fand sich ein Krystall, dessen Endflächen genügend gut ausgebildet waren, um entscheidende Messungen vornehmen zu können. Die Prismenzone der Krystalle zeigte immer starke Streifung und Endflächen waren nur selten zu beobachten.

Klinoklas konnte nur auf wenigen Stufen wahrgenommen werden und auch dann nur in mikroskopischen Krystallen. Zu seiner Bestimmung dienten seine blauschwarze Farbe und charakteristische Lötrohrproben.

Konichalcit wurde sehr spärlich in den charakterislischen, smaragdgrünen kugeligen Formen gefunden.

Andere Kupfermineralien wurden in sehr geringen Mengen beobachtet; einige derselben konnten nicht mit Sicherheit mit irgend einer der bekannten Mineralspecies identificiert werden, die vorkommenden Mengen waren aber viel zu gering, um eine eingehende Untersuchung zu gestatten.

Mineral. Laborat. der Sheffield Scientific School of Yale University, New Haven, Connecticut; im Februar 1910. 